\title{
CT scans in psychiatric patients - an exploratory study at Chris Hani Baragwanath Hospital
}

F Y Jeenah, MMed (Psych)

M Y H Moosa, MMed (Psych), FC (Psych)

Division of Psychiatry, Department of Neurosciences, University of the Witwatersrand, Johannesburg

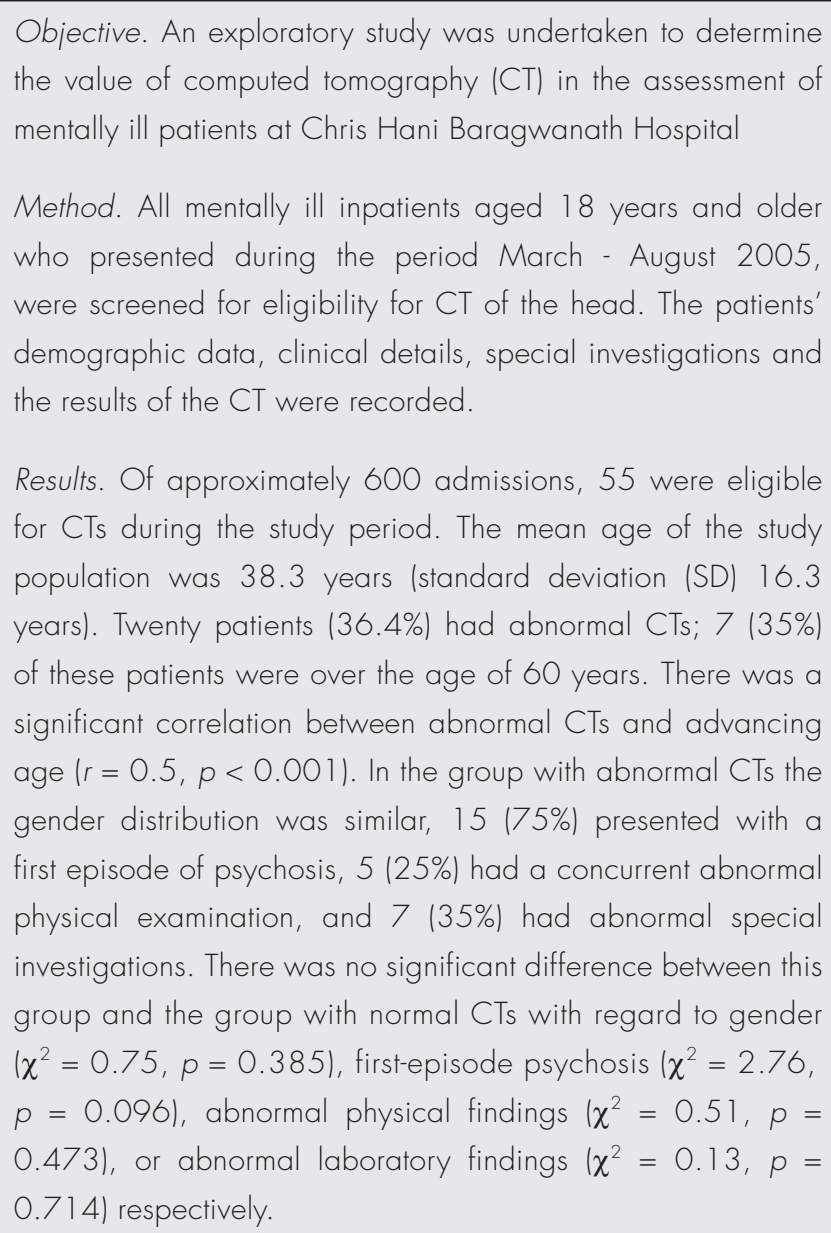

Results. Of approximately 600 admissions, 55 were eligible for CTs during the study period. The mean age of the study population was 38.3 years (standard deviation (SD) 16.3 years). Twenty patients (36.4\%) had abnormal CTs; 7 (35\%) of these patients were over the age of 60 years. There was a significant correlation between abnormal $C T$ s and advancing age $(r=0.5, p<0.001)$. In the group with abnormal $C T_{s}$ the gender distribution was similar, $15(75 \%)$ presented with a first episode of psychosis, 5 (25\%) had a concurrent abnormal physical examination, and 7 (35\%) had abnormal special investigations. There was no significant difference between this group and the group with normal CTs with regard to gender $\left(\chi^{2}=0.75, p=0.385\right)$, first-episode psychosis $\left(\chi^{2}=2.76\right.$, $p=0.096)$, abnormal physical findings $\left(\chi^{2}=0.51, p=\right.$ $0.473)$, or abnormal laboratory findings $\left(\chi^{2}=0.13, p=\right.$ $0.714)$ respectively.

Conclusion. In this psychiatric population CT scanning revealed a significant number of abnormalities, especially in patients with first-episode psychosis. The study also suggests that clinical abnormalities (physical and laboratory) may not be reliable predictors of abnormal CTs and therefore the need for further research with bigger sample size.

The introduction of computed tomography (CT) is recognised as one of the most important innovations in the recent history of clinical medicine. The value of this non-invasive technique in examining intracranial contents was quickly realised in the diagnosis and detection of vascular accidents and tumours.

In psychiatric research studies, CT provides important insights into the aetiology and long-term structural abnormalities in the brains of patients with schizophrenia, mood disorders, metabolic and neurological disorders of the brain. However, the place of CT in clinical psychiatry is less clear. Disorders of the central nervous system resulting from trauma, intracranial haemorrhage, tumours and vascular abnormalities, etc. may initially manifest as psychiatric symptoms. As these symptoms may be misdiagnosed as indicating a primary psychiatric disorder, there is a danger of missing potentially treatable causes if a CT scan of the brain is not performed.

Although the use of CT has been advocated for various conditions, there are no guidelines for its use in psychiatric patients. However, its use can be divided in two groups as follows: (i) to confirm organic illness, inferring that definitive physical illness such as seizure disorder, stroke, and alcohol/drug use can mimic psychiatric disorders; and (ii) as a preventive measure to screen all psychiatric patients, similar to the use of blood tests and urinalysis.' Current literature provides little evidence on the definitive role of CT as a diagnostic tool in psychiatry. International and local studies ${ }^{2.7}$ report indications for CT in psychiatric patients as being confusion and/or dementia of unknown cause, first episode of a psychosis of unknown aetiology, movement disorder of unknown aetiology, prolonged catatonia, first episode of major affective disorder, personality change after 50 years of age, and the presence of cognitive decline. Some psychiatrists recommend CT for all first admissions to hospital. ${ }^{2}$

In South Africa, more than 50\% of illnesses presenting in tertiary institutions are due to communicable diseases and injury. A significant percentage of these patients present with predominantly psychiatric symptoms and may warrant CT. Chris Hani Baragwanath Hospital in Soweto has a psychiatric unit with facilities for 155 inpatients and regularly admits acutely ill patients who fit this profile and who warrant CT of the head as part of their assessment. Because of the high cost of this procedure and the limited resources, it is important to have evidence-based guidelines for eligibility for CT. This study was an exploratory study to provide further information on this procedure when creating guidelines. 


\section{Objectives}

Objectives of the study were as follows: (i) to determine the yield of abnormal scans using a set of criteria for eligibility for CT and to describe these abnormalities; and (ii) to describe the demographic and clinical characteristics of patients with abnormal scans and to determine the differences, if any, between this group and the group with normal scans.

\section{Method}

This prospective study was conducted at Chris Hani Baragwanath Hospital in Soweto. All mentally ill inpatients aged 18 years and older who presented during the period March - August 2005 were screened, and those who underwent CT of the head were included in the study.

\section{Procedure}

The study was approved by the Human Research Ethics Committee (HREC) of the University of the Witwatersrand. The procedure was fully explained to each patient and written consent was obtained. If the patient was eligible for CT but did not have the capacity to understand and give written consent, this was obtained from alternative sources (family or hospital superintendent). The patients' demographic data lage, race and gender), clinical details (physical, mental state) and all other special investigations conducted (laboratory, radiological and EEG) were recorded by the investigators. Thereafter the patients underwent $\mathrm{CT}$ and the scan was read by a radiologist who was blind to the patients' history and initial diagnosis.

\section{Criteria for eligibility}

The following patients were eligible for CT scanning: (i) all those presenting with a first episode of psychosis (with or without mood features); and (ii) all psychotic patients (with or without mood features) with either features of a delirium, some focal physical or neurological signs, and/or abnormal results of special investigations.

\section{Statistical analysis}

Descriptive statistics were computed as counts and percentages, minimum and maximum, and means (standard deviations (SD), 95\% confidence intervals $(\mathrm{Cls})$. The chi-square $\left(\chi^{2}\right)$ test was used to determine the relationship between general categorical characteristics lage group, sex and clinical and laboratory findings) in the groups with abnormal and normal CTs.
Correlations were done using Pearson's correlation co-efficient for age and abnormality of CT. All analyses were done using Statistical Package for Social Sciences 10.0 for Windows (SPSS Inc., Chicago, III.). A value of $p<0.05$ was considered significant.

\section{Results}

During this 6-month period approximately 600 patients were screened and a total of 55 were found to be eligible for X-ray CT. All 55 patients gave written consent and were included in the study. Twenty of these patients (36.4\%) showed some abnormal findings, and the details are listed in Table I.

\begin{tabular}{ll} 
Table I. Abnormal CT findings $(\mathbf{N}(\%))$ & N \\
\hline Details & 1 \\
\hline Trauma: Blow-out fracture of the orbits & 6 \\
Vascular: Old infarct with/without calcification & 6 \\
Global cerebral atrophy & 7 \\
Mass lesion: Pituitary adenoma; & 6 \\
TB granuloma; neurocysticercosis & \\
\hline
\end{tabular}

The mean age of the study population was 38.3 years (SD 16.3, 95\% Cl: 33.9 - 42.7, minimum 18, maximum 73 years). Seven patients (35\%) with abnormal CT were over the age of 60 years compared with only 1 patient in the group with a normal scan $\left(\chi^{2}=17.2, p=0.0006\right)$. There was also a significant correlation between abnormal CT and advancing age $(r=0.5, p<0.001)$ (Fig. 1).

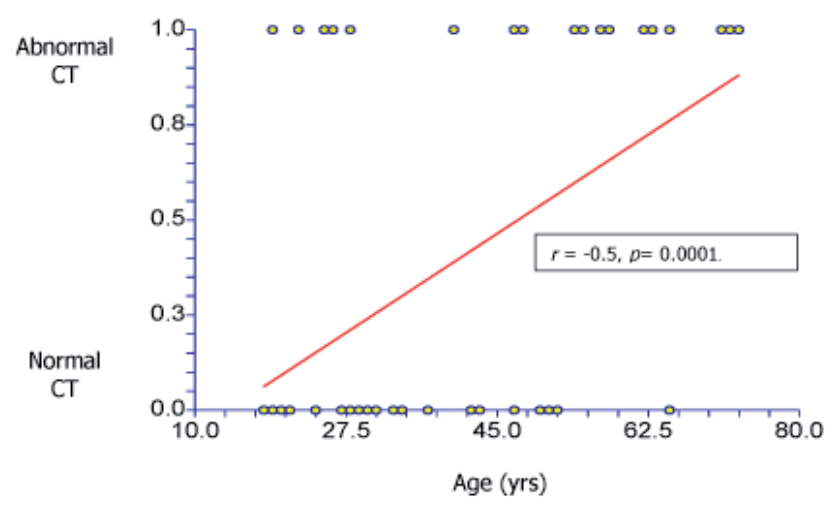

Fig. 1. Correlation between abnormal X-ray CTs and age.

In the group with abnormal CTs the gender distribution was similar, $15(75 \%)$ presented with a first episode of psychosis, 5 (25\%) had a concurrent abnormal physical examination (blindness, tardive dyskinesia, chronic alcoholism, diabetes mellitus), and 7 (35\%) had abnormal special investigations (blood, cerebrospinal (CSF) 


\section{articles}

and HIV tests, and EEG). There was no significant difference between this group and the group with normal CTs with regard to gender $\left(\chi^{2}=0.75, p=0.385\right)$, firsteepisode psychosis $\left(\chi^{2}=\right.$ $2.76, p=0.096)$, abnormal physical findings $\left(\chi^{2}=0.51, p=\right.$ $0.473)$, or abnormal laboratory findings $\left(\chi^{2}=0.13, p=0.714\right)$ respectively (Table II).

Only 2 of the patients with abnormal CTs had a first episode of psychosis and either some focal physical or neurological signs and/or abnormal results of special investigations.

\section{Discussion}

In this study the yield of abnormal scans was significantly high (36.4\%). Similar high yields have been reported in other studies: $86 \%$ of 37 patients $^{8}$ and $57 \%$ of 323 patients. ${ }^{9}$ In contrast, Agzarian et al..$^{10}$ reported that $95 \%$ of 397 patients at an acute tertiary service were within normal limits and McClellan et al." reported that $88.1 \%$ of all inpatients during a 3 -year period were within normal limits. Both studies with low yields reported that in the few patients with abnormal findings the main finding was cortical atrophy. We suggest that if scanning is done routinely the yield would be considerably lower; however, if as in this study the patients were screened adequately, the yield would probably be higher.

A significantly large percentage of our patients with abnormal, CTs were over the age of 60 years and there was a positive correlation between abnormal CTs and advancing age. Similar high yields of abnormal CTs in firsteepisode psychosis patients in this age group have been reported in other studies. ${ }^{12}$

The factor most predictive of abnormal CT in our patients was a first episode of psychosis $132 \%$ of all patients with firstepisode psychosis, and $85 \%$ of all patients with abnormal scans). Previous studies $^{2,13,14}$ also recommend that all patients presenting with a first onset of psychotic illness should receive CT of the head to rule out such causes as tumours, abscesses, Huntington's disease, encephalitis, Wilson's disease, and trauma. Yet other studies ${ }^{15}$ suggest that scanning is indicated if there is an atypical presentation or inadequate response to standard treatment in such patients.

Some researchers ${ }^{11,12,16}$ recommend that only mentally ill patients with neurological abnormalities (namely focal signs, seizures, head injuries, etc.) and/or abnormal special investigations (EEG, blood tests etc. $\left.\right|^{4}$ should be eligible for $\mathrm{CT}$. This is similar to the guidelines of the Chris Hani Baragwanath unit. However, in our group of patients only a significant minority of the patients with abnormal CTs had a concurrent abnormal physical examination and/or special investigations. This suggests that if we use these criteria for scanning strictly, we will most probably miss many abnormalities. This view is supported by Colohan et al, ${ }^{17}$ who also report a poor correlation between abnormalities on $\mathrm{CT}$ scan and findings on physical examination, laboratory testing, EEG and psychological testing. It is recommend that in firstepisode

\begin{tabular}{|c|c|c|c|}
\hline Characteristics & Abnormal scans $(N=20)$ & Normal scans $(N=35)$ & Statistical analysis \\
\hline \multicolumn{4}{|l|}{ Age group (yrs) } \\
\hline $18-30$ & $6(30.0)$ & 19 (54.3) & \\
\hline $31-45$ & $1 \quad(5.0)$ & $11(31.4)$ & \\
\hline $46-60$ & $6(30.0)$ & $4(11.4)$ & \\
\hline$>60$ & $7(35.0)$ & $1(2.9)$ & $\chi^{2}=17.2, p=0.0006$ \\
\hline \multicolumn{4}{|l|}{ Gender } \\
\hline Male & $9(45.0)$ & $20(57.1)$ & \\
\hline Female & $11(55.0)$ & $15(42.9)$ & $\chi^{2}=0.75, p=0.385$ \\
\hline \multicolumn{4}{|l|}{ Clinical findings } \\
\hline First episode psychosis & $15(75)$ & $32(91.4)$ & $\chi^{2}=2.76, p=0.096$ \\
\hline $\begin{array}{l}\text { Abnormal physical } \\
\text { examination }\end{array}$ & $5(25.0)$ & $12(34.3)$ & $\chi^{2}=0.51, p=0.473$ \\
\hline $\begin{array}{l}\text { Abnormal special } \\
\text { investigations }\end{array}$ & $7(35.0)$ & $14(40.0)$ & $\chi^{2}=0.13, p=0.714$ \\
\hline Blood investigations & $3(15.0)$ & $3(8.6)$ & \\
\hline HIV-positive & $1(5.0)$ & $6(17.1)$ & \\
\hline CSF investigation & $1(5.0)$ & $3(8.6)$ & \\
\hline EEG & $0(0.0)$ & $0(0.0)$ & \\
\hline \multicolumn{4}{|c|}{ CSF = cerebrospinal fluid; EEG = electro-encephalogram. } \\
\hline
\end{tabular}


psychosis, clinical and laboratory findings should be used either for clarification or to complement other findings, ' and that utilising all of these factors to guide the ordering of CTs would greatly increase the yield of the procedure for psychiatric patients, without excess medical morbidity. ${ }^{5}$

This study is limited in its generalisability because the study population were attendees of a tertiary academic hospital and represent a select group of severely ill patients. The small sample size may have statistical limitations in terms of ascertaining meaningful differences when comparing groups. Notwithstanding these limitations the findings were significant and different from published data and warrant further investigation into the role of CT scanning in mentally ill patients.

\section{Conclusion}

The study revealed that CT scans in this psychiatric population yielded a significant number of abnormalities, especially in patients with first-episode psychosis. This study also suggests that clinical abnormalities (physical and laboratory) may not be reliable predictors of abnormal CTs. It is recommended that further studies using a larger sample size be conducted to determine if guidelines should include all patients with first-episode psychosis despite the high cost and limited availability of this procedure.

\section{References}

1. Ananth J, Gamal R, Miller M, et al. Is the routine CT head scan justified for psychiatric patients? A prospective study. J Psychiatry Neurosci 1993; 18: 69-73

2. Weinberger DR. Brain disease and psychiatric illness: when should a psychiatrist order a CAT scan? Am J Psychiatry 1984; 141: 1521-1527.

3. Hollister LE, Boutros N. Clinical use of CT and MR scans in psychiatric patients. J Psychiatry Neurosci 1991; 16: 194-198.

4. Hollister LE, Shah NN. Structural brain scanning in psychiatric patients: a further look. Clin Psychiatry 1996; 57: 241-244

5. Moles JK, Franchina لJ, Sforza PP. Increasing the clinical yield of computerized tomography for psychiatric patients. Gen Hosp Psychiatry 1998: 20: 282-291.

6. Berk $M$. Indications for computed tomographic brain scanning in psychiatric inpatients. S Afr Med J 1992; 82: 338-340.

7. Olfson M. Utilization of neuropsychiatric diagnostic tests for general hospital patients with mental disorders. Am J Psychiatry 1992: 149: 171 1-1717.

8. Chandler JD, Patten J. Head CT in new geriatric psychiatry patients: a prospective study. J Geriatr Psychiatry Neurol 1989; 2: 101-105

9. Roberts JK, Lishman WA. The use of the CAT head scanner in clinnical psychiatry. Br J Psychiatry 1984; 145: 152-158

10. Agzarian M, Chryssidis S, Davies RP, et al. Use of routine computed tomography brain scanning of psychiatry patients. Australas Radiol 2006; 50: 27-28.

11. McClellan RL, Eisenberg RL, Giyanani VL. Routine CT screening of psychiatric inpatients. Radiology 1988; 169: 99-100.

12. Tsai I, Tsuang MT. How can we avoid unnecessary CT scanning for psychiatric patients? J Clin Psychiatry 1981; 42: 452-454

13. Gewirtz G, Squires-Wheeler E, Sharif Z, et al. Results of computed tomography during first admission for psychosis. Br J Psychiatry 1994; 164: 789-795.

14. Battaglia J, Spector IC. Utility of the CAT scan in a first psychotic episode. Gen Hosp Psychiatry 1988; 10: 398-40 1.

15. Pickuth D, Heywang-Kobrunner SH, Spielmann RP. Routine skull CT in psychiatric diagnosis. Radiologe 1999; 39: 995-999.

16. Larson EB, Mack LA, Watts B, et al. Computed tomography in patients with psychiatric illnesses: advantage of a 'rule in' approach. Ann Intern Med 1981; 95: 360-364

17. Colohan $\mathrm{H}, \mathrm{O}^{\prime}$ Callaghan $\mathrm{E}$, Larkin $\mathrm{C}$, et al. An evaluation of cranial $\mathrm{CT}$ scanning in clinical psychiatry. Ir J Med Sci 1989; 158: 178-181 